\title{
THE GALACTIC BLACK HOLE TRANSIENT H1743-322 DURING OUTBURST DECAY: CONNECTIONS BETWEEN TIMING NOISE, STATE TRANSITIONS, AND RADIO EMISSION
}

\author{
E. Kalemci, ${ }^{1,2}$ J. A. Tomsick, ${ }^{3}$ R. E. Rothschild, ${ }^{3}$ K. Pottschmidt, ${ }^{3}$ S. Corbel ${ }^{4}$ and P. Kanet ${ }^{5}$ \\ Received 2005 July 8; accepted 2005 October 28
}

\begin{abstract}
Multiwavelength observations of Galactic black hole transients during outburst decay are instrumental for our understanding of the accretion geometry and the formation of outflows around black hole systems. H1743-322, a black hole transient observed intensely in X-rays and also covered in the radio band during its 2003 decay, provides clues about the changes in accretion geometry during state transitions and also the general properties of X-ray emission during the intermediate and low-hard states. In this work, we report on the evolution of spectral and temporal properties in X-rays and the flux in the radio band, with the goal of understanding the nature of state transitions observed in this source. We concentrate on the transition from the thermal dominant state to the intermediate state that occurs on a timescale of 1 day. We show that the state transition is associated with a sudden increase in power-law flux. We determine that the ratio of the power-law flux to the overall flux in the $3-25 \mathrm{keV}$ band must exceed 0.6 for us to observe strong timing noise. Even after the state transition, once this ratio was below 0.6, the system transited back to the thermal dominant state for 1 day. We show that the emission from the compact radio core does not turn on during the transition from the thermal dominant state to the intermediate state but does turn on when the source reaches the low-hard state, as seen in $4 \mathrm{U} 1543-47$ and GX 339-4. We find that the photon index correlates strongly with the QPO frequency and anticorrelates with the rms amplitude of variability. We also show that the variability is more likely to be associated with the power-law emission than the disk emission.
\end{abstract}

Subject headings: accretion, accretion disks — binaries: close — black hole physics stars: individual (H1743-322) - X-rays: binaries - X-rays: stars

\section{INTRODUCTION}

The Galactic black hole transients show several correlated spectral and temporal variability properties during outbursts, denoted as spectral states. During the initial rise and at the end of the decay before quiescence, these transients are usually in the "low-hard" state (LHS). In this state, a hard power-law component dominates the $\mathrm{X}$-ray spectrum, and strong variability ( $>20 \%$ rms amplitude) and quasi-periodic oscillations (QPOs) are often observed. In between the rise and the decay, the source may evolve through a combination of "thermal dominant" and "steep power-law" states. In the thermal dominant state (TDS), the soft disk component dominates the spectrum, and the timing noise is very low or absent. In the steep power-law state, the power-law flux in the $2-20 \mathrm{keV}$ band accounts for more than $50 \%$ of the flux and has a photon index $(\Gamma)$ greater than 2.4 . Moderate variability and QPOs are observed in this state. There also exist intermediate states (IS) in which source characteristics do not fit into the steep power law, TDS, or LHS, but show various combinations of these states (see McClintock \& Remillard 2006 for detailed discussion of spectral states). Throughout this work, we use the name IS for the particular intermediate state between the TDS and the LHS during the outburst decay. Even though these states were historically characterized using X-ray observations, changes in other bands occur as well. In the TDS,

\footnotetext{
${ }^{1}$ Space Sciences Laboratory, 7 Gauss Way, University of California, Berkeley, CA 94720-7450.

${ }^{2}$ Current address: Sabancı University, Orhanlı-Tuzla 34956, İstanbul, Turkey.

${ }^{3}$ Center for Astrophysics and Space Sciences, Code 0424, University of California at San Diego, La Jolla, CA 92093-0424.

${ }^{4}$ AIM-Unité Mixte de Recherche CEA-CNRS-Université Paris VII-UMR 7158, CEA Saclay, Service d'Astrophysique, F-91191 Gif sur Yvette, France.

5 Department of Physics and Astronomy, University of Iowa, Van Allen Hall, Iowa City, IA 52242.
}

the radio emission from the compact core is quenched (Fender et al. 1999; Corbel et al. 2000). Optically thin outflows are sometimes detected during state transitions (Fender \& Kuulkers 2001; Corbel et al. 2001), and powerful, compact jets are always observed in the LHS (Fender 2001). The optical and infrared emission also show state-dependent properties (Kalemci et al. 2005; Homan et al. 2005a; Corbel \& Fender 2002).

The multiwavelength observations made during the decaying portion of the outbursts provide valuable information about black hole transients because of the very high probability of observing transitions from the TDS to the IS, and eventually to the LHS (Kalemci 2002). The changes during the transitions can reveal the geometry and physical environment of these systems before and after the transitions (Esin et al. 1997; Zdziarski et al. 2002). The LHS contains additional information due to strong variability and strong radio emission, both correlating with spectral parameters. Our group has been observing these transients during outburst decay in X-rays with the Rossi X-Ray Timing Explorer $(R X T E)$ and in radio to understand the evolution before, during, and after the state transitions (Kalemci et al. 2001, 2003, 2005; Tomsick et al. 2001, 2003). Our emphasis is on state transitions, and especially on understanding the changes in X-rays while the radio jet is turning on. A uniform analysis of all black hole transients observed with approximately daily coverage with $R X T E$ during outburst decay between 1996 and 2001 provided important information on the evolution of spectral and temporal parameters during the decay (Kalemci et al. 2004a). The sharpest change indicating a state transition is observed to be a jump in the rms amplitude of variability from less than a few percent to more than tens of percent in less than 1 day. This change in the rms amplitude is almost always accompanied by a sharp increase in the powerlaw flux. There is also evidence that the strong rms noise is only observed when the power-law flux from the source is above a 
certain percentage of the total flux. This sharp change in rms amplitude of variability is noted as the time of state transition from the TDS to a harder state in Kalemci et al. (2004a), and the same definition is applied here. During the outburst decay, the photon index, disk temperature, and disk flux usually decrease slowly. Often, late in the outburst, the disk flux becomes undetectable. After the transition, characteristic frequencies of the power-density spectrum also decrease with time.

H1743-322 was discovered with the Ariel 5 (Kaluzienski \& Holt 1977) and HEAO 1 (Doxsey et al. 1977) satellites in 1977 August. After a couple of detections in 1984 with EXOSAT (Reynolds 1999), and in 1996 with the TTM/COMIS telescope on Mir-Kvant (Emelyanov et al. 2000), the source was detected in outburst again in 2003 March with INTEGRAL (Revnivtsev et al. 2003) and RXTE (Markwardt \& Swank 2003). The radio (Rupen et al. 2003a), infrared (Baba et al. 2003), and optical (Steeghs et al. 2003) counterparts were quickly identified during the 2003 outburst. The radio observations revealed relativistic jets $(v / c \simeq 0.8$; Rupen et al. 2004b; Corbel et al. 2005). Largescale jets were also detected in X-rays with Chandra at the end of the outburst (Corbel et al. 2005). The X-ray observations with RXTE and INTEGRAL indicate that the source went through several spectral states before fading at the end of 2003 (Markwardt \& Swank 2003; Homan et al. 2003; Kretschmar et al. 2003; Grebenev et al. 2003; Tomsick \& Kalemci 2003; Parmar et al. 2003; Joinet et al. 2005). Even though there is no mass measurement of the compact object, the X-ray spectral and temporal properties and a high-frequency QPO pair with frequencies similar to those of other black hole sources (Homan et al. 2005b) establish this source as a very likely black hole.

In this work we characterize the X-ray and radio properties of H1743-322 during the outburst decay in 2003, compare these properties to the general properties of black hole transients, and discuss the unique properties of this source in detail. We especially concentrate on the triggering mechanism for the state transitions during the outburst decay.

\section{OBSERVATIONS AND ANALYSIS}

\subsection{The RXTE Observations}

The RXTE All-Sky Monitor (ASM) light curve of H1743322 in the 2003 outburst is shown in Figure 1. Our daily monitoring campaign with RXTE (under observation ID 80137, each pointing 2-3 ks long) started on MJD 52,906 after the source's ASM count rate dropped below 15 counts $\mathrm{s}^{-1}$. The source was in the TDS until MJD 52,930, when a transition to the IS occurred (Tomsick \& Kalemci 2003). After MJD 52,938, the source was in the LHS (Kalemci et al. 2004b). In the LHS, we also obtained longer exposure observations (10-15 ks; see Table 1) to investigate spectral and temporal properties of the source in greater detail than the daily monitoring observations. The monitoring program has very good coverage of the transitions and evolution during the outburst decay (see Fig. 1). Table 1 shows the list of the observations we used in this work.

\subsubsection{X-Ray Spectral Analysis}

For most of the observations, we used both the Proportional Counter Array (PCA) and the High-Energy X-Ray Timing Experiment (HEXTE) instruments on RXTE for the spectral analysis (see Bradt et al. [1993] for instrument descriptions). For the PCA, the $3-25 \mathrm{keV}$ band was used, and the response matrix and background model were created using the standard FTOOLS (version 5.3.1) programs. We added $0.8 \%$ up to $7 \mathrm{keV}$ and $0.4 \%$ above $7 \mathrm{keV}$ as systematic error (for the details of how we esti-

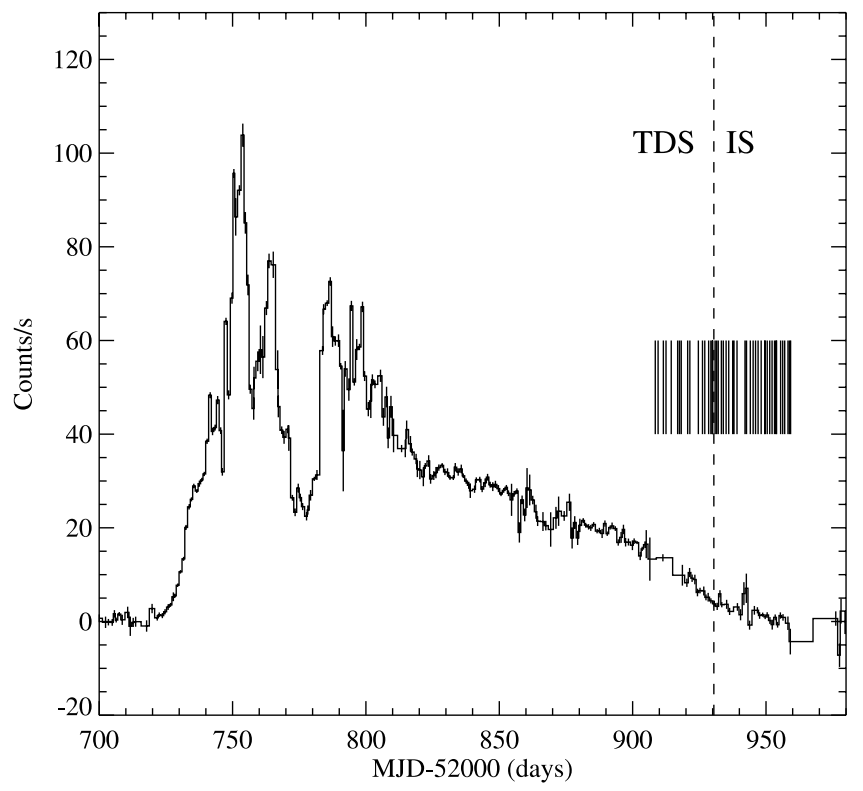

FIG. 1.-The 2-12 keV ASM light curve of the 2003 outburst of H1743-322. The vertical lines indicate the times for $R X T E$ pointings that we analyzed. The dashed line indicates the approximate time of state transitions from the TDS to the IS.

mated systematic uncertainties, see Tomsick et al. 2001). We used all available proportional counter units (PCUs) for each observation, choosing the combination that would give the maximum number of counts per pointing.

The 15-200 keV band was used for the HEXTE data. We used the response matrix created by FTOOLS and applied the necessary dead-time correction (Rothschild et al. 1998). The HEXTE background is measured throughout the observation by alternating between the source and background fields every $32 \mathrm{~s}$. As H1743 -322 is close to the plane $\left(b=-1^{\circ} .83\right)$, both the source confusion and the Galactic ridge emission are important for determining the HEXTE background. By using the HEXTEROCK utility and Galactic bulge scans and comparing the rates between "positive" and "negative" off-source cluster positions, we determined that for both clusters, positive background pointing provides a better estimate of the background for our observations. For cluster A, the negative pointing is in the Galactic plane, with very strong ridge contribution, and background sources are contributing in the cluster $\mathrm{B}$ negative position. The relative normalization between the PCA and the HEXTE is kept free, and varies between 0.9 and 1 . The HEXTE data were included in the spectral analysis until MJD 52,950. After this date, including HEXTE data did not improve the fits due to low fluxes and short observation times.

The Galactic ridge emission was a factor for all of the observations discussed here because of the proximity of the source to the Galactic plane. We determined that our last nine observations in 2004 (MJD 53,021-53,055) had a constant flux that represented the ridge emission with a power-law index of 2.325 and an iron line at $6.62 \mathrm{keV}$. The $3-25 \mathrm{keV}$ unabsorbed flux from the ridge emission is $1.08 \times 10^{-10} \mathrm{ergs} \mathrm{cm}^{-2} \mathrm{~s}^{-1}$. These numbers are consistent with expectations from the Galactic ridge (Revnivtsev 2003). To make sure, we also analyzed the $R X T E$ Galactic bulge scans before and after the outburst. We determined that the model described above fits them as well. For all observations, we fixed the ridge parameters as given above. After MJD 52,960, our monitoring observations did not detect the source significantly above the Galactic ridge emission. 
TABLE 1

Observational Parameters

\begin{tabular}{|c|c|c|c|c|c|c|c|c|}
\hline $\begin{array}{l}\text { Observation } \\
\text { No. }^{\text {a }}\end{array}$ & $\begin{array}{l}\text { Date } \\
(\mathrm{MJD})\end{array}$ & $\begin{array}{l}\text { Exposure }^{\mathrm{b}} \\
(\mathrm{ks})\end{array}$ & $\Gamma$ & $T_{\text {in }}$ & PL Flux ${ }^{c}$ & DBB Flux ${ }^{d}$ & $\begin{array}{l}\mathrm{rms} \\
(\%)^{\mathrm{e}}\end{array}$ & Notes \\
\hline $01-00 \ldots \ldots \ldots . . .$. & 52908.49 & 2.40 & $2.73 \pm 0.07$ & $0.82 \pm 0.01$ & 5.06 & 24.63 & $<3.25$ & \\
\hline $02-00 \ldots \ldots \ldots \ldots$ & 52909.53 & 3.00 & $2.40 \pm 0.05$ & $0.81 \pm 0.01$ & 6.03 & 23.86 & $<3.25$ & \\
\hline $03-00 \ldots \ldots \ldots . .$. & 52911.52 & 3.54 & $2.24 \pm 0.03$ & $0.80 \pm 0.01$ & 8.34 & 23.00 & $<3.23$ & \\
\hline 04-00 ............ & 52912.50 & 3.54 & $2.18 \pm 0.03$ & $0.800 \pm 0.01$ & 10.4 & 22.11 & $2.17 \pm 1.48$ & \\
\hline $05-00 \ldots \ldots \ldots . .$. & 52914.47 & 2.40 & $2.30 \pm 0.04$ & $0.780 \pm 0.01$ & 4.91 & 19.87 & $1.66 \pm 0.99$ & \\
\hline $11-00 \ldots \ldots \ldots \ldots$ & 52916.88 & 3.48 & $2.25 \pm 0.03$ & $0.76 \pm 0.01$ & 7.33 & 16.69 & $2.78 \pm 2.10$ & \\
\hline $07-00 \ldots \ldots \ldots . . .$. & 52917.56 & 3.48 & $2.18 \pm 0.03$ & $0.74 \pm 0.01$ & 6.95 & 15.84 & $2.24 \pm 1.11$ & \\
\hline 08-00 ........... & 52918.21 & 2.94 & $2.25 \pm 0.04$ & $0.75 \pm 0.01$ & 6.66 & 15.53 & $1.28 \pm 1.33$ & \\
\hline 09-00 ............ & 52920.62 & 2.52 & $2.36 \pm 0.05$ & $0.74 \pm 0.01$ & 3.50 & 14.02 & $1.86 \pm 0.80$ & \\
\hline $10-00 \ldots \ldots \ldots . .$. & 52921.44 & 2.28 & $2.35 \pm 0.09$ & $0.73 \pm 0.01$ & 3.47 & 12.57 & $2.28 \pm 1.16$ & \\
\hline $13-00 \ldots \ldots \ldots . .$. & 52924.62 & 2.70 & $2.31 \pm 0.07$ & $0.70 \pm 0.01$ & 3.20 & 10.32 & $<2.04$ & Radio (VLA) observation with no detection. \\
\hline $14-00 \ldots \ldots \ldots . .$. & 52926.18 & 3.48 & $2.29 \pm 0.05$ & $0.68 \pm 0.01$ & 3.70 & 8.65 & $<2.53$ & \\
\hline $15-00 \ldots \ldots \ldots . .$. & 52927.04 & 3.42 & $2.24 \pm 0.06$ & $0.68 \pm 0.01$ & 2.97 & 8.03 & $<2.15$ & \\
\hline $16-00 \ldots \ldots \ldots . .$. & 52928.48 & 1.86 & $2.18 \pm 0.07$ & $0.66 \pm 0.01$ & 3.48 & 6.96 & $<2.80$ & Galactic ridge $\sim 10 \%$ of the overall flux. \\
\hline $17-00 \ldots \ldots \ldots . .$. & 52929.32 & 3.54 & $2.01 \pm 0.05$ & $0.66 \pm 0.01$ & 4.05 & 6.38 & $<2.03$ & \\
\hline $18-00 \ldots \ldots \ldots . .$. & 52929.91 & 1.56 & $2.14 \pm 0.05$ & $0.65 \pm 0.01$ & 4.58 & 5.87 & $<1.37$ & \\
\hline $18-01 \ldots \ldots \ldots . .$. & 52929.98 & 2.04 & $2.07 \pm 0.05$ & $0.65 \pm 0.01$ & 4.44 & 5.92 & $<1.77$ & Still in the TD state. \\
\hline $19-01 \ldots \ldots \ldots . .$. & 52930.90 & 1.56 & $2.20 \pm 0.04$ & $0.64 \pm 0.01$ & 8.17 & 4.96 & $7.18 \pm 0.27$ & Timing noise, transition to the IS. \\
\hline $19-00 \ldots \ldots \ldots . .$. & 52931.46 & 1.98 & $2.27 \pm 0.03$ & $0.63 \pm 0.01$ & 8.18 & 4.83 & $7.60 \pm 0.52$ & \\
\hline $20-00 \ldots \ldots \ldots \ldots$ & 52932.07 & 3.30 & $2.22 \pm 0.02$ & $0.62 \pm 0.01$ & 11.66 & 3.25 & $11.15 \pm 0.46$ & $\begin{array}{l}\text { No radio detection at MJD } 52932.96 . \\
\text { QPO at } 7.8 \mathrm{~Hz} \text {. }\end{array}$ \\
\hline $21-00 \ldots \ldots \ldots .$. & 52933.27 & 3.42 & $2.10 \pm 0.05$ & $0.62 \pm 0.01$ & 4.63 & 4.11 & $1.85 \pm 0.26$ & $\begin{array}{l}\text { Timing noise reduces substantially for } \\
\text { this observation only! }\end{array}$ \\
\hline $22-00 \ldots \ldots \ldots . .$. & 52933.99 & 3.48 & $2.23 \pm 0.02$ & $0.60 \pm 0.01$ & 8.77 & 3.06 & $10.35 \pm 1.10$ & QPO at $7.6 \mathrm{~Hz}$. \\
\hline $23-00 \ldots \ldots \ldots . .$. & 52935.04 & 2.88 & $2.25 \pm 0.03$ & $0.58 \pm 0.01$ & 6.07 & 2.89 & $9.77 \pm 1.36$ & QPO at $7.1 \mathrm{~Hz}$. \\
\hline $24-00 \ldots \ldots \ldots . .$. & 52936.02 & 2.28 & $2.20 \pm 0.02$ & $0.58 \pm 0.01$ & 7.88 & 2.12 & $11.44 \pm 0.56$ & QPO at $7.7 \mathrm{~Hz}$. \\
\hline $25-00 \ldots \ldots \ldots \ldots$ & 52937.51 & 3.48 & $2.17 \pm 0.02$ & $0.55 \pm 0.02$ & 9.47 & 1.26 & $15.71 \pm 0.91$ & QPO at $6.9 \mathrm{~Hz}$. \\
\hline $26-00 \ldots \ldots \ldots . .$. & 52938.00 & 2.10 & $2.06 \pm 0.02$ & $0.52 \pm 0.03$ & 9.61 & 0.80 & $21.22 \pm 1.13$ & $\begin{array}{l}\text { LHS according to McClintock \& Remillard } \\
\text { (2006). QPO at } 5.9 \mathrm{~Hz} \text {. }\end{array}$ \\
\hline $27-00 \ldots \ldots \ldots . .$. & 52939.12 & 2.04 & $2.07 \pm 0.02$ & $0.50 \pm 0.03$ & 8.58 & 0.67 & $19.47 \pm 0.78$ & Radio (VLA) detection! QPO at $5.8 \mathrm{~Hz}$. \\
\hline $28-00 \ldots \ldots \ldots . .$. & 52942.12 & 1.98 & $1.86 \pm 0.02$ & $0.50 \pm 0.08$ & 6.31 & 0.20 & $26.21 \pm 1.08$ & QPO at $2.9 \mathrm{~Hz}$. \\
\hline $29-00 \ldots \ldots \ldots . .$. & 52942.68 & 1.62 & $1.87 \pm 0.03$ & $0.59 \pm 0.11$ & 5.66 & 0.19 & $19.82 \pm 2.34$ & QPO at $2.4 \mathrm{~Hz}$. \\
\hline L01-00 ......... & 52944.10 & 10.08 & $1.78 \pm 0.01$ & $0.42 \pm 0.05$ & 4.91 & 0.13 & $27.51 \pm 2.63$ & QPO at $1.8 \mathrm{~Hz}$. \\
\hline $30-00 \ldots \ldots \ldots . .$. & 52945.17 & 1.80 & $1.78 \pm 0.02$ & $0.45 \pm 0.06$ & 4.02 & 0.14 & $26.46 \pm 3.69$ & \\
\hline L01-01 .......... & 52946.07 & 10.62 & $1.69 \pm 0.02$ & $0.47 \pm 0.07$ & 3.59 & 0.09 & $28.96 \pm 2.54$ & \\
\hline $31-00 \ldots \ldots \ldots . .$. & 52947.02 & 1.08 & $1.79 \pm 0.03$ & $0.39 \pm 0.10$ & 2.80 & 0.06 & $29.93 \pm 5.11$ & \\
\hline $32-00 \ldots \ldots \ldots . .$. & 52948.19 & 5.22 & $1.78 \pm 0.02$ & $0.29 \pm 0.02$ & 2.29 & 0.03 & $30.11 \pm 2.23$ & \\
\hline $33-00 \ldots \ldots \ldots .$. & 52949.53 & 3.54 & $1.74 \pm 0.02$ & $0.37 \pm 0.07$ & 1.99 & 0.05 & $36.40 \pm 2.41$ & Last radio (VLA) detection. \\
\hline $\mathrm{L} 01-02 \ldots \ldots \ldots$. & 52949.67 & 10.38 & $1.76 \pm 0.01$ & $0.28 \pm 0.01$ & 1.79 & 0.03 & $39.29 \pm 5.45$ & \\
\hline $34-00 \ldots \ldots \ldots . .$. & 52950.44 & 2.04 & $1.78 \pm 0.03$ & $0.36 \pm 0.06$ & 1.47 & 0.03 & $32.27 \pm 12.20$ & Only PCA is used from this observation on. \\
\hline $35-00^{\mathrm{f}} \ldots \ldots \ldots$ & 52951.39 & 3.72 & $1.74 \pm 0.03$ & $0.37 \pm 0.06$ & 1.02 & 0.04 & $\ldots$ & Galactic ridge is as strong as the source. \\
\hline $36-00 \ldots \ldots \ldots . .$. & 52952.26 & 1.56 & $1.86 \pm 0.04$ & $0.29 \pm 0.03$ & 0.84 & 0.01 & $\ldots$ & \\
\hline $\mathrm{L} 02-00 \ldots \ldots \ldots$ & 52953.18 & 14.28 & $1.83 \pm 0.02$ & $0.28 \pm 0.02$ & 0.58 & 0.02 & $\ldots$ & \\
\hline $\mathrm{L} 02-01 \ldots \ldots . . .$. & 52953.69 & 3.96 & $1.83 \pm 0.05$ & $0.30 \pm 0.04$ & 0.49 & 0.02 & $\ldots$ & \\
\hline $\mathrm{L} 02-02 \ldots \ldots \ldots$ & 52953.96 & 8.76 & $1.72 \pm 0.05$ & $0.40 \pm 0.03$ & 0.41 & 0.04 & $\ldots$ & \\
\hline $37-00 \ldots \ldots \ldots . .$. & 52955.46 & 1.74 & $1.98 \pm 0.22$ & $0.38 \pm 0.08$ & 0.18 & 0.03 & $\ldots$ & \\
\hline $38-00 \ldots \ldots \ldots . .$. & 52956.26 & 3.06 & $1.71 \pm 0.20$ & $0.35 \pm 0.06$ & 0.13 & 0.02 & $\ldots$ & Radio (ATCA) observation with no detection. \\
\hline $39-00 \ldots \ldots \ldots . .$. & 52957.00 & 1.44 & $2.43 \pm 0.29$ & $0.36 \pm 0.09$ & 0.09 & 0.02 & $\ldots$ & \\
\hline $40-00 \ldots \ldots \ldots . .$. & 52958.23 & 3.66 & $2.32 \pm 0.25$ & $0.33 \pm 0.06$ & 0.08 & 0.02 & $\cdots$ & \\
\hline $\mathrm{L} 03-0 \mathrm{x}^{\mathrm{g}} \ldots \ldots$. & 52958.82 & 12.12 & $2.51 \pm 0.66$ & $0.35 \pm 0.05$ & 0.02 & 0.02 & $\ldots$ & \\
\hline L03-01 ......... & 52959.21 & 14.64 & $1.79 \pm 0.41$ & $0.36 \pm 0.03$ & 0.03 & 0.02 & $\ldots$ & \\
\hline
\end{tabular}

a Full observation ID is, e.g., 80137-01-Obs for observations that do not start with L, and 80137-02-Obs for those that start with L, denoting "long."

b Exposure time.

c Unabsorbed power-law flux in the $3-25 \mathrm{keV}$ band, in units of $10^{-10} \mathrm{ergs} \mathrm{cm}^{-2} \mathrm{~s}^{-1}$

d Unabsorbed disk-blackbody flux in the $3-25 \mathrm{keV}$ band, in units of $10^{-10} \mathrm{ergs} \mathrm{cm}^{-2} \mathrm{~s}^{-1}$.

e rms amplitude of variability in the $3-30 \mathrm{keV}$ band after ridge correction.

${ }^{f}$ Observations 34-01, 35-00, and 35-01 were merged.

g Observations L03-00, L03-02, and L03-03 were merged. 
For all the observations, our first spectral model consisted of absorption (phabs in XSPEC), smeared edge (smedge in XSPEC; Ebisawa et al. 1994), a multicolor disk blackbody (diskbb in XSPEC; Makishima et al. 1986), a power law (pegpwrlw in XSPEC), a narrow Gaussian to model the iron line, and the ridge emission (as described above). This model has been commonly used for the spectral analysis of black holes in the LHS (Tomsick $\&$ Kaaret 2000; Sobczak et al. 2000; Kalemci et al. 2005). The hydrogen column density was fixed to $N_{\mathrm{H}}=2.3 \times 10^{22} \mathrm{~cm}^{-2}$ based on the Chandra results (Miller et al. 2004). The smeared edge width was fixed to $10 \mathrm{keV}$. Once we fit the observations with this model, we added a high-energy cutoff (highecut in XSPEC) to the model. None of the observations showed a significant decrease in $\chi^{2}$, which would indicate a high-energy cutoff.

\subsubsection{X-Ray Temporal Analysis}

For each observation, we computed the power density spectra (PDS) from the PCA data using IDL programs developed at the University of Tübingen (Pottschmidt 2002) for three energy bands, $3-6 \mathrm{keV}, 6-15 \mathrm{keV}$, and $15-30 \mathrm{keV}$. We also computed the PDS for the combined band of 3-30 keV. The source flux above $30 \mathrm{keV}$ is too low for timing analysis. The PDS was normalized as described in Miyamoto \& Kitamoto (1989) and corrected for the dead-time effects according to Zhang et al. (1995), with a dead time of $10 \mu$ s per event. Using $256 \mathrm{~s}$ time segments, we investigated the low-frequency QPOs and the timing properties of the continuum up to $256 \mathrm{~Hz}$ for different energy bands. We fit all our PDSs with broad and narrow Lorentzians with our standard timing analysis techniques (Kalemci et al. 2005; Kalemci 2002; Pottschmidt 2002).

In the PDS fits, Lorentzians with quality value (centroid frequency divided by the width) $Q>2$ are denoted as QPOs. The rms amplitudes are calculated over a frequency band from zero to infinity. We multiplied the rms amplitude of variability with $T^{2} /[T-(R+B)]^{2}$, where $T$ is the overall count rate, $B$ is the background rate determined using pcabackest, and $R$ is the count rate due to the Galactic ridge, to obtain the variability inherent to the source (Berger \& van der Klis 1994).

\subsection{Radio Observations}

We obtained Very Large Array (VLA) radio information (observations conducted by M. Rupen) for dates between MJD 52,920 and 52,949. The "core" was not detected in 4.86 and $8.64 \mathrm{GHz}$ frequencies on MJD 52,924.9 and 52,933.0. Another radio component (from an earlier discrete plasma ejection) away from the core was detected during this time (Rupen et al. 2004c). The first detection of the core during the decay occurred on MJD $52,940.0$, with a flux density of $0.14 \pm 0.04 \mathrm{mJy}^{\text {beam }}{ }^{-1}$ (M. Rupen 2003, private communication). The last detection of the core with the VLA occurred on MJD 52,949, with a flux density of $0.22 \pm 0.04 \mathrm{mJy}$ at $4.86 \mathrm{GHz}$ (Rupen et al. 2004a).

Toward the end of the 2003 outburst, the source was observed with the Australia Telescope Compact Array (ATCA). The source was not detected on MJD 52,955.86, 52,973.72, 52,983.45, $52,994.49$, or $53,049.40$, with the highest rms value of $0.10 \mathrm{mJy}$ at 4.8 GHz (Corbel et al. 2005). The evolution of the radio fluxes during the outburst decay is given in Figure $2 b$.

\section{RESULTS}

We investigated the evolution of several spectral and temporal fit parameters during the decay of H1743-322 to establish the time of state transitions. Some of the important parameters are

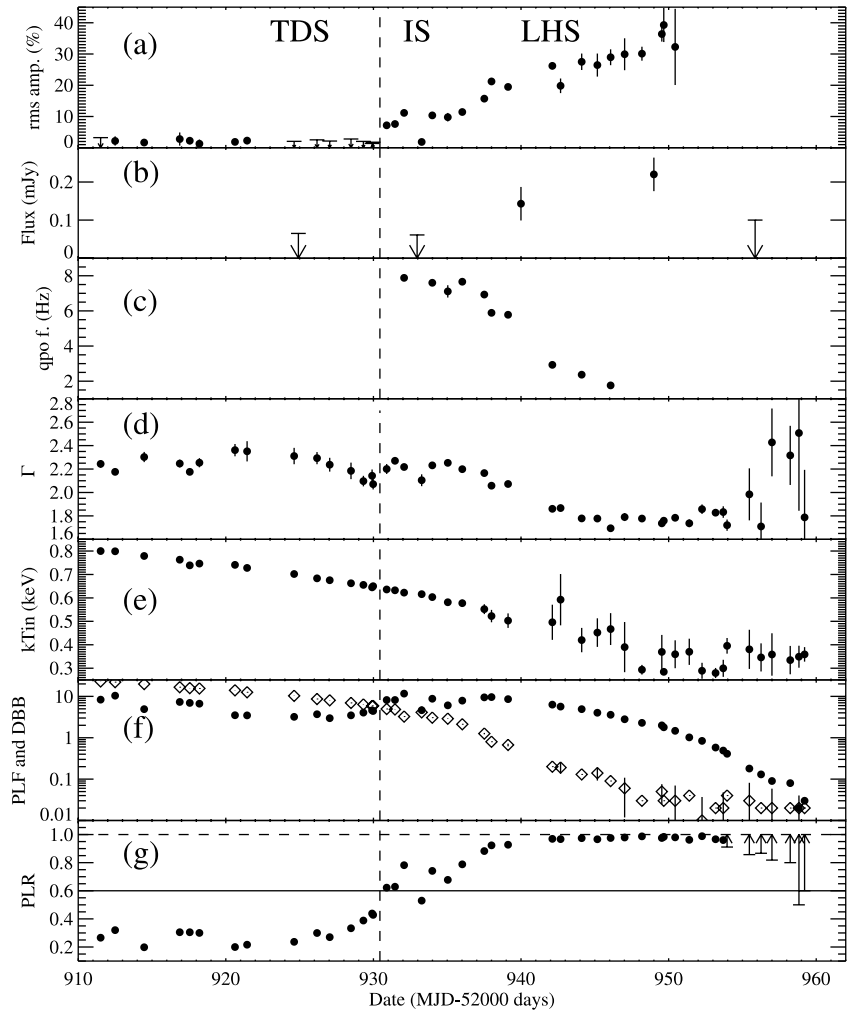

FIG. 2. - Evolution of ( $a$ ) the total rms amplitude of variability in the $3-30 \mathrm{keV}$ band, $(b)$ the radio flux density at $4.86 \mathrm{GHz},(c)$ the QPO frequency, $(d)$ the photon index $(\Gamma),(e)$ the inner disk temperature $T_{\text {in }},(f)$ the power-law flux (circles) and the disk-blackbody flux (diamonds) in the 3-25 keV band in units of $10^{-10} \mathrm{ergs} \mathrm{cm}^{-2}$ $\mathrm{s}^{-1}$, and $(g)$ the ratio of the power-law flux to the total flux in the 3-25 keV band. The dashed line indicates the case for which all the emission comes from the power-law component. The solid line shows the threshold for observing timing noise. Unabsorbed fluxes are shown. For most of the measurements, the $1 \sigma$ uncertainties are smaller than the plot symbols. The vertical dashed line indicates the approximate time of transition from the TDS to the IS. The first four data points in $(b)$ are from VLA, and the final point is from ATCA.

shown in Figure 2, and some of the spectral fit parameters are tabulated in Table 1.

\subsection{States and Transitions}

Until MJD 52,930, the spectral and temporal properties of the source indicated a TDS. When the noise was detected in the PDS, it was characterized by a weak power law typically observed in the TDS (see Fig. 3a). The rms amplitude of variability was less than 3\%. On MJD 52,930, the power-law flux doubled, while the power-law ratio (PLR, the ratio of the power-law flux to the total flux in the $3-25 \mathrm{keV}$ band) exceeded 0.6, and the PDS showed detectable timing noise, with an rms amplitude of $\sim 7 \%$. The spectral and temporal properties (see Figs. $3 b$ and $3 d$ ) indicate an IS right after this transition. During the transition, the photon index increased slightly. The evolutions of $T_{\text {in }}$ and the disk-blackbody (DBB) flux were smooth.

A QPO appeared on MJD 52,932 as the PLR reached 0.8. Just after this date, for one observation, the power-law flux and the PLR dropped. The strong timing signature also disappeared, and the PDS only showed a weak power-law component. The source properties were similar to those of the TDS. This single observation is called "TD-like" for this reason. Within half a day, the power-law flux increased to the IS levels, and the timing noise reappeared, along with the QPO (see Fig. 3). Based on the TDS observations and the TD-like observation, we set a PLR threshold 


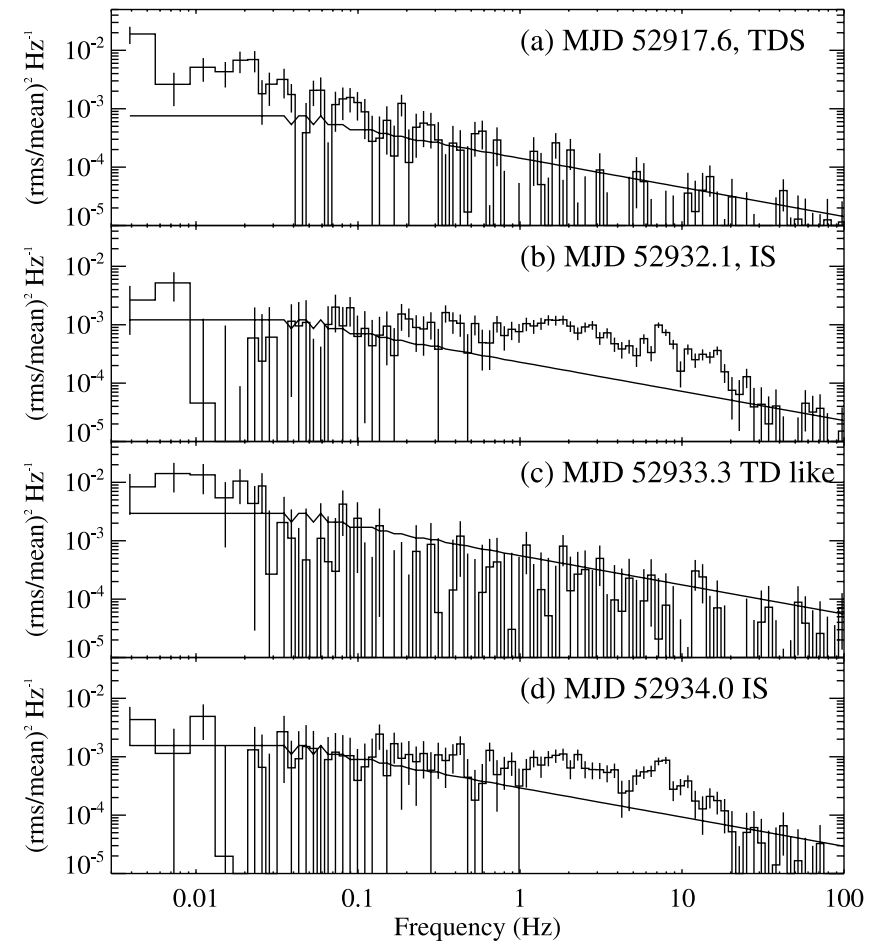

FIG. 3.- Power spectra ( $a$ ) in the TDS, $(b)$ after the transition to the IS, $(c)$ during the TD-like observation, and $(d)$ right after the TD-like observation, again in the IS PDSs in $(b),(c)$, and $(d)$ are approximately 1 day apart. The solid line shows the effective Poisson noise level (uncertainty in Poisson noise subtraction after merging segments and binning the data; Nowak et al. 1999).

of variability of $\sim 0.6$ for H1743-322. The duration for the TD-like case is of the order of 1 day. Interestingly, the photon index decreased during the TD-like observation, returning to the value measured right before the transition to the IS.

QPOs with decreasing frequency were observed in 10 observations after the transition. After MJD 52,933, the rms amplitude of variability increased, the photon index hardened, and the inner disk temperature and DBB flux decreased smoothly. At the end of this evolution, the source was in the LHS. The transition to the LHS was smooth, and it is difficult to determine when the source left the IS. Based on the McClintock \& Remillard (2006) criteria (spectrum dominated by the power-law component, $1.5<\Gamma<$ 2.1 , rms amplitude of variability $>10 \%$ ), the transition happened around MJD 52,938. In the LHS the photon index hovered between 1.7 and 1.9 until MJD 52,956. The rms amplitude of variability increased until MJD 52,950. After this date, the statistical quality of the data was not good enough to determine the rms amplitude of variability.

\subsection{Correlations between Spectral and Temporal Parameters}

It has been well established that the black hole transients show several correlations between spectral and temporal parameters (Kalemci 2002, and references therein). During outburst decays, the photon index shows the strongest correlations (or anticorrelations) with the QPO frequency and the rms amplitude of variability (Vignarca et al. 2003; Kalemci 2002). We plotted these parameters as a function of photon index in Figure 4. We also included the PLR as a function of photon index, as it shows a strong correlation with the rms amplitude of variability in the IS. Figure 4 shows that the rms amplitude of variability is strongly anticorrelated (linear correlation coefficient of -0.949), and the QPO frequency is strongly correlated (linear correlation coefficient of 0.987 ) with the photon index. Notice that there might be

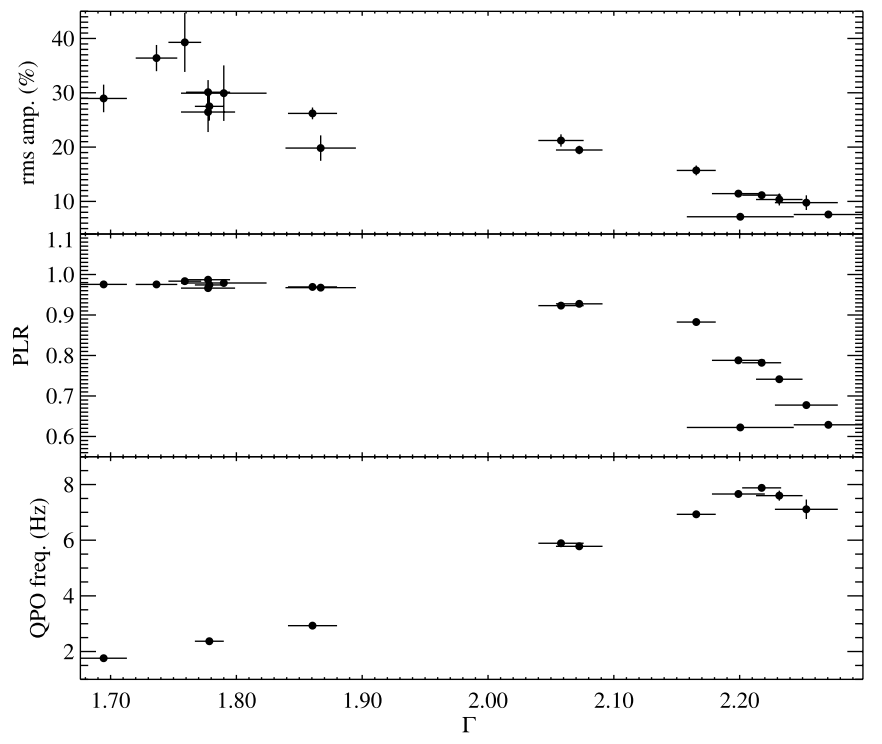

FIG. 4.-Correlation between the photon index and the rms amplitude of variability (top), the PLR (middle), and the QPO frequency (bottom) for observations taken between MJD 52,930.9 and 52,949.7, excluding the TD-like observation.

a turnover (or a saturation) in the QPO- $\Gamma$ relation, as seen earlier for other sources by Vignarca et al. (2003). The TD-like observation is not shown. Obviously, it does not obey the anticorrelation between the rms amplitude of variability and $\Gamma$.

Another interesting relation is between the rms amplitude of variability and the disk flux. It is well known that the rms amplitude of variability increases with energy for systems with significant disk emission (Kalemci 2002), but once this disk component is not significant, the relation between rms amplitude of variability and energy is not predictable (Kalemci et al. 2004a). In Figure 5, we show the rms amplitude of variability for two energy bands, their ratio, and the PLR. The presence of DBB emission clearly reduces the rms amplitude of variability in the $3-6 \mathrm{keV}$

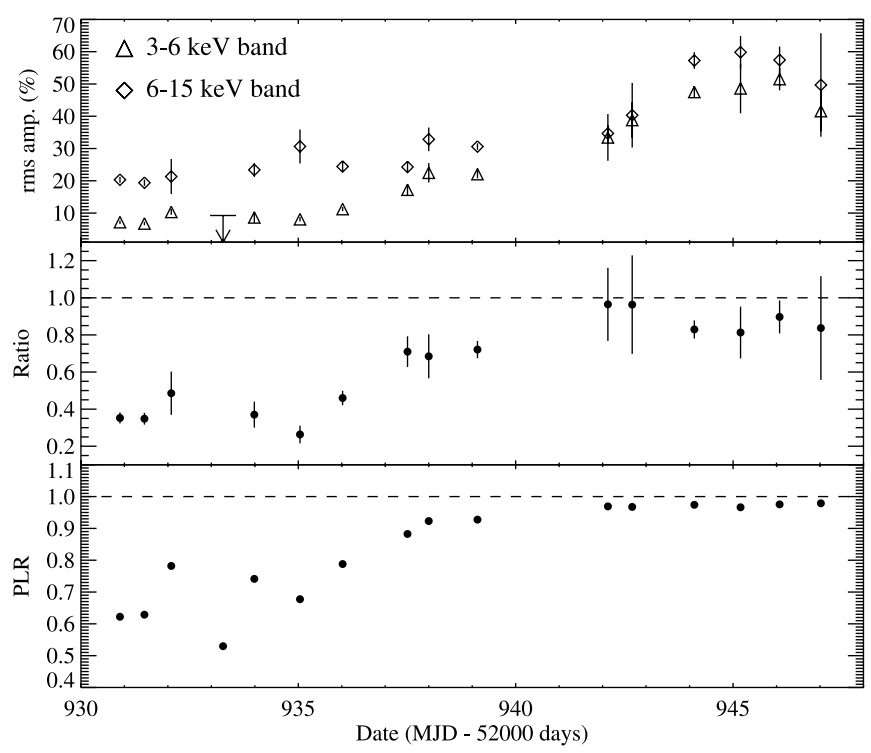

FIG. 5.-Top: Evolution of the rms amplitude of variability in the $3-6 \mathrm{keV}$ (triangles) and 6-15 keV (diamonds) bands. The $3 \sigma$ upper-limit rms amplitude of variability for the TD-like observation in the $6-15 \mathrm{keV}$ band $(9.25 \%)$ is shown with an arrow. The $3-6 \mathrm{keV}$ band upper limit of $3.28 \%$ is not shown for clarity. Middle: The ratio of the rms variability in the $3-6 \mathrm{keV}$ and $6-15 \mathrm{keV}$ bands. Bottom: The PLR. 
band. For most of the observations during which the DBB emission is not significant, the ratio between rms amplitudes of variability is consistent with unity. But when the last six observations are grouped, it is clear that the $6-15 \mathrm{keV}$ band rms amplitude of variability is higher than that of the $3-6 \mathrm{keV}$ band.

\section{DISCUSSION}

\subsection{Evolution of Spectral and Temporal Properties during Outburst Decay}

In general, the evolution of the spectral and temporal parameters of H1743-322 show very similar characteristics to the evolution of other black hole systems during outburst decay. The transition from the TDS to the IS is sharp, and is marked by a jump in the rms amplitude of variability, the power-law flux, and the PLR (Kalemci et al. 2004a, 2005). We note that, during the transition, both the power-law flux and the PLR increase substantially. But the sudden increase in the PLR is always due to a sudden increase in the power-law flux, and not to a sudden decrease in DBB emission. Therefore, the physical change that causes the state transition is associated with a sudden increase in the powerlaw flux. On the other hand, the PLR provides a threshold for strong variability. After the transition from the TDS to the IS, the DBB flux and inner disk temperature steadily decrease, and finally the source reaches the LHS.

A detailed discussion of several scenarios that could result in such evolution during the decay is given for $4 \mathrm{U}$ 1543-47 in Kalemci et al. (2005). H1743-322 shows spectral and temporal evolution in X-rays quite similar to those of $4 \mathrm{U} 1543-47$. In addition, for both cases, the radio core was only detected after the sources reached their hardest levels, and not detected right after the transition to the IS. For H1743-322, the fits never require a high-energy cutoff in the spectrum, while for $4 \mathrm{U} 1543-47$, a cutoff is observed only during the transition to the LHS. It is possible that a portion of electrons are nonthermal for both sources, at least in the TDS and IS (Coppi 1999). For H1743-322, the evolution of $T_{\text {in }}$ is consistent with an accretion disk receding away from the black hole (and the drop in QPO frequency may also be an indication of this). The evolutions of the PLR and $\Gamma$ indicate a Comptonizing medium (either an independent corona or the base of a jet) getting stronger as the decay progresses. The lack of adequate multiwavelength coverage does not allow us to strongly constrain the details of different models, as we were able to do for $4 \mathrm{U} 1543-47$.

Even though most of the properties of H1743-322 are generic to the black hole transients during decay, it also showed two unexpected behaviors. The first one is the TD-like observation on MJD 52,932. Once the transition to a harder state occurs, it is unusual for a system to go back to the previous, softer state just for 1 day during the outburst decay. (Short branchings to harder states are common, such as branching seen in the 1998 outburst of XTE J1550-564; Homan et al. 2001). For the 14 outburst decays analyzed in Kalemci 2002, only XTE J1859+226 showed a similar behavior in its outburst decay in 1999. After the transition to the IS at MJD 51,524, XTE J1859+226 transited back to the TDS twice for a short time (Kalemci 2002). A recent report indicates that during the decay of the 2002-2003 outburst of GX 339-4, the source transited back to the TDS for 3 days after the transition to the IS (Belloni et al. 2005). We note that these branchings with timescales of a few days may be more common, as frequent monitoring is required to catch them. If we examine the cases source by source, rather than outburst by outburst, from the seven transients that have been observed with daily monitoring, three sources showed this behavior. The IS is a transi- tional state between the TDS and the LHS, but these branchings imply that the evolution is not monotonic. Transitions back and forth to the TDS can occur on a timescale of days. In contrast, once the source is in the hard state and the X-ray luminosity keeps decreasing, the process is irreversible; there is no example (to the best of our knowledge) of a transition back to the IS or the TDS.

A second unexpected behavior is the slight increase in photon index ( $\sim 6 \%$ ) during the transition to the IS (see Fig. 2). The only other case that showed an increase in photon index during a transition from the TDS to the IS is the 2000 outburst of XTE J1550564 (Kalemci et al. 2004a; Rodriguez et al. 2003). During such transitions, the power-law index usually does not change, or decreases somewhat (Kalemci et al. 2004a). For both H1743-322 and XTE J1550-564, the power-law flux and the PLR showed a sudden increase, while the photon index also increased. However, for H1743-322, this transition resulted in an increase in rms amplitude of variability, whereas for XTE J1550-564, it resulted in a decrease of rms amplitude of variability. Note that the conditions for these two transitions were not the same. For H1743322 , the transition was from the TDS to an IS, whereas for XTE J1550-564, the transition was from a soft IS to a harder IS, en route to the LHS.

\subsection{Emission Mechanisms during the State Transition from the TDS to the IS}

In the TDS, the bulk of the emission comes from the disk as multicolor blackbody radiation. The evolution of the DBB component during the transition to the IS is smooth. On the other hand, the power-law component in the X-ray spectrum shows a sudden change, and is perhaps associated with the state transition. For the power-law component, the two important observations are (1) a sudden (less than 1 day) and strong (100\%) increase in the flux, and (2) a small change (6\% increase) in the photon index. We note that an increase in the power-law flux with only little or no change in the power-law index is a universal property of the state transitions that coincide with the appearance of timing noise and QPOs for most of the black hole systems (Kalemci et al. 2004a). In this section, we discuss various scenarios that may explain the evolution of the power-law component during the transition to the IS.

For thermal Comptonization, the photon index depends on two parameters of the corona: the temperature and the optical depth of the Comptonizing medium (Sunyaev \& Titarchuk 1980). The power-law flux depends on the input soft flux, the optical depth, and the covering fraction of the Comptonizing medium. Increasing the soft input flux would result in a larger power-law flux and also may cause a lower coronal temperature due to cooling. Our observations do not indicate an increase in the DBB flux. A sudden expansion of the corona (while keeping the optical depth constant) could explain the change in power-law flux if the volume increase corresponds to an increase in the covering fraction. Before the transition, the rms amplitude of variability is less than $1.8 \%$; therefore, there is at least a factor of 4 increase in the rms amplitude of variability, whereas there is only a factor of 2 increase in the power-law flux. Therefore, the volume increase should have a nonlinear relation to the variability amplitude to explain the strong increase. Emergence of another soft photon source inside the corona (with a spectrum not contributing in the PCA range) is another possible explanation for the transition. One candidate is synchrotron radiation inside the corona, causing synchrotron self-Compton emission (Markoff \& Nowak 2004). In this case, the hard power-law emission would be created by a combination of thermal Comptonization of synchrotron and disk photons and nonthermal synchrotron self-Compton emission. For the case 
of synchrotron, the seed photons come from inside, while for the DBB, the seed photons come from both the outside and the inside of the corona, depending on the assumed geometry. It is possible that the strong variability is created more efficiently if the source is inside the corona. Since the major change is not related to the temperature and optical depth of the corona, it could also explain why the photon index varies little during the transition. The small change in the index may be due to additional cooling (if the increase in the secondary soft flux is more than the decrease in the DBB flux), or the effect of additional nonthermal Comptonization. If the strong variability in the IS and the LHS is due to an additional soft component such as the synchrotron radiation, then the origin of low-frequency QPOs will not be the accretion disk (Lee \& Miller 1998; Titarchuk \& Fiorito 2004). One can also envision a combination of scenarios, such as an expansion of the corona while the synchrotron self-Compton contribution is increasing.

Another way to increase the power-law flux is to have two emission mechanisms producing power-law spectra. During the transition, the second component, which also produces the timing noise, may become active. One possibility is that during the TDS the bulk motion (dynamical) Comptonization (Laurent \& Titarchuk 1999; Titarchuk \& Fiorito 2004) may be the main mechanism, whereas in the IS, thermal Comptonization starts to operate. This requires that both components produce very similar power-law spectra right at the transition. The secondary component in the IS may also be direct synchrotron emission from a jet (Falcke \& Biermann 1999; Markoff et al. 2001). This, again, requires that both emission mechanisms produce a similar spectrum at the transition. There is another argument against this second possibility. The radio observations indicate that the emission from the compact jet began sometime between MJD 52,933 and 52,940, well after the transition to the IS. (Since there was no optical or infrared monitoring of the source during the transition, we were not able to pinpoint the time of jet formation.) Based on the comparison between the optical and IR light curves and the X-ray and radio properties of GX 339-4 (Homan et al. 2005a) and 4U 1543-47 (Kalemci et al. 2005), one can argue that the compact jet does not turn on before the source is settled in the LHS. H1743322 seems to support this argument, as the compact radio jet was first detected after the source was in the LHS, according to McClintock \& Remillard (2006). We note that, during the rise, a compact radio core was observed, even after the transition from the LHS to the IS (Joinet et al. 2005; Rupen et al. 2003). This shows that, even though the formation of the compact jet requires the LHS with almost no soft emission in the PCA band, it can be sustained for a while in the presence of soft emission (Corbel et al. 2004).

\subsection{The Correlations}

There is a very strong correlation between the photon index and the QPO frequency up to a photon index of 2.2. At high photon indices, the relation seems to be turning over. XTE J1550-564 and GRO J1655-40 are two other sources that show a turnover in the QPO frequency-photon index relation (Vignarca et al. 2003). Relativistic effects may cause this turnover, if the QPO has an "accretion ejection instability" origin (Varnière et al. 2002; Vignarca et al. 2003). We note that we cannot conclusively claim that the relation turns over for H1743-322, because the evidence for a turnover is based on a single data point (see Fig. 4). A saturation QPO frequency with photon index could be a sign of a system dominated by a converging inflow (Titarchuk \& Fiorito 2004).

There is a strong anticorrelation between the photon index and the rms amplitude of variability. This is a global relation that is valid for most black hole transients during outburst decay
(Kalemci et al. 2003). One can also observe a strong correlation between the PLR and the rms amplitude of variability at high spectral indices (see Fig. 4). This shows the diminishing effect of the DBB radiation on the rms amplitude of variability. Another way to investigate the effect of DBB on the rms amplitude of variability is to compare the energy dependence of variability. Figure 5 shows that for the observations with strong DBB emission, the rms amplitude of variability is much stronger in the higher energy band than in the lower energy band. One can see a correlation between the ratio of the rms amplitude of variability in two bands and the PLR. Once the DBB emission is negligible (PLR $\sim 1$ ), the relation between the rms amplitude of variability and energy is not that clear. The correlations between the PLR and both the rms amplitude of variability and the variability ratio indicate that the variability is generated in the power-law component, and that the DBB component regulates the rms amplitude of variability by changing the mean flux (Churazov et al. 2001; Kalemci 2002).

\section{SUMMARY AND CONCLUSIONS}

We analyzed the $R X T E$ X-ray observations of H1743-322 during its outburst decay in 2003. In addition to the X-ray observations, we also obtained radio fluxes and discussed their evolution with respect to the state transitions. The evolution of the spectral and temporal fit parameters show similar properties to those of other sources. The transition from the TDS to the IS is marked by a strong increase in the rms amplitude of variability and power-law flux. This transition may correspond to the emergence of a secondary soft component, such as synchrotron radiation, or a secondary power-law component. Future multiwavelength observations of these transients may help in making the distinction. At the time of this transition, the radio core was not detected. Three days after the transition, the source went back to the TDS for 1 day, indicating that the IS is not stable. After the source returned to the IS, it gradually reached the low-hard state. The core is detected in the radio band when the X-ray spectrum is totally dominated by the power-law emission.

There is a strong correlation between the photon index and the QPO frequency. At high QPO frequencies and photon indices, the correlation shows a turnover (or saturates). The threshold PLR for the state transition and the correlation between the PLR and the ratio of rms amplitude of variability in the $6-15 \mathrm{keV}$ and 3-6 keV bands show that the DBB emission dilutes the amplitude of variability. When the DBB emission is absent, the rms amplitude of variability in two bands is similar. This may indicate that the origin of the variability and the QPO is the corona itself, rather than the disk.

E. K. acknowledges NASA grant NAG5-13142 and the partial support of TÜBITAK. E. K. thanks all scientists who contributed to the Tübingen Timing Tools. The authors thank Michael Rupen for the valuable VLA data. E. K. thanks Lev Titarchuk for his remarks on state transition and evolution. This work made use of the Galactic bulge scans of Craig Markwardt. J. A. T. acknowledges partial support from NASA grant NNG04GB19G. R. E. R. acknowledges NASA grant NAS5-30720. P. K. acknowledges partial support from a University of Iowa Faculty Scholar Award. The authors also thank the anonymous reviewer for his comments, which significantly improved the scientific quality of the paper. The Australia Telescope is funded by the Commonwealth of Australia for operation as a national facility managed by CSIRO. 
Baba, D., Nagata, T., Iwata, I., Kato, T., \& Yamaoka, H. 2003, IAU Circ., 8112, 2 Belloni, T., Homan, J., Casella, P., van der Klis, M., Nespoli, E., Lewin, W. H. G., Miller, J. M., \& Mendez, M. 2005, A\&A, 440, 207

Berger, M., \& van der Klis, M. 1994, A\&A, 292, 175

Bradt, H. V., Rothschild, R. E., \& Swank, J. H. 1993, A\&AS, 97, 355

Churazov, E., Gilfanov, M., \& Revnivtsev, M. 2001, MNRAS, 321, 759

Coppi, P. S. 1999, in ASP Conf. Ser. 161, High Energy Processes in Accreting Black Hole, ed. J. Poutanen \& R. Svensson (San Francisco: ASP), 375

Corbel, S., \& Fender, R. P. 2002, ApJ, 573, L35

Corbel, S., Fender, R. P., Tomsick, J. A., Tzioumis, A. K., \& Tingay, S. 2004, ApJ, 617, 1272

Corbel, S., Fender, R. P., Tzioumis, A. K., Nowak, M., McIntyre, V., Durouchoux, P., \& Sood, R. 2000, A\&A, 359, 251

Corbel, S., Kaaret, P., Fender, R. P., Tzioumis, A. K., Tomsick, J. A., \& Orosz, J. A. $2005, A p J, 632,504$

Corbel, S., et al. 2001, ApJ, 554, 43

Doxsey, R., et al. 1977, IAU Circ., 3113, 1

Ebisawa, K., et al. 1994, PASJ, 46, 375

Emelyanov, A. N., Aleksandrovich, N. L., \& Sunyaev, R. A. 2000, Astron. Lett., 26, 297

Esin, A. A., McClintock, J. E., \& Narayan, R. 1997, ApJ, 489, 865

Falcke, H., \& Biermann, P. L. 1999, A\&A, 342, 49

Fender, R., et al. 1999, ApJ, 519, L165

Fender, R. P. 2001, MNRAS, 322, 31

Fender, R. P., \& Kuulkers, E. 2001, MNRAS, 324, 923

Grebenev, S. A., Lutovinov, A. A., \& Sunyaev, R. A. 2003, Astron. Tel., 189

Homan, J., Buxton, M., Markoff, S., Bailyn, C., Nespoli, E., \& Belloni, T. 2005a, ApJ, 624, 295

Homan, J., Miller, J. M., Wijnands, R., Steeghs, D., Belloni, T., van der Klis, M., \& Lewin, W. H. G. 2003, Astron. Tel., 162

Homan, J., Miller, J. M., Wijnands, R., van der Klis, M., Belloni, T., Steeghs, D., \& Lewin, W. H. G. 2005b, ApJ, 623, 383

Homan, J., Wijnands, R., van der Klis, M., Belloni, T., van Paradijs, J., Klein-Wolt, M., Fender, R., \& Méndez, M. 2001, ApJS, 132, 377

Joinet, A., Jourdain, E., Malzac, J., Roques, J. P., Schönfelder, V., Ubertini, P., \& Capitanio, F. 2005, ApJ, 629, 1008

Kalemci, E. 2002, Ph.D. thesis, Univ. California, San Diego

Kalemci, E., Tomsick, J. A., Buxton, M. M., Rothschild, R. E., Pottschmidt, K., Corbel, S., Brocksopp, C., \& Kaaret, P. 2005, ApJ, 622, 508

Kalemci, E., Tomsick, J. A., Rothschild, R. E., Pottschmidt, K., Corbel, S., Wijnands, R., Miller, J. M., \& Kaaret, P. 2003, ApJ, 586, 419

Kalemci, E., Tomsick, J. A., Rothschild, R. E., Pottschmidt, K., \& Kaaret, P. 2001, ApJ, 563, 239 2004a, ApJ, 603, 231 2004b, in AIP Conf.

Proc. 714, X-ray Timing 2003: Rossi and

Beyond, ed. P. Kaaret, F. K. Lamb, \& J. H. Swank (New York: AIP), 52
Kaluzienski, L. J., \& Holt, S. S. 1977, IAU Circ., 3106, 1

Kretschmar, P., Chenevez, J., Capitanio, F., Orr, A., Palumbo, G., \& Grebenev, S. 2003, Astron. Tel., 180

Laurent, P., \& Titarchuk, L. 1999, ApJ, 511, 289

Lee, H. C., \& Miller, G. S. 1998, MNRAS, 299, 479

Makishima, K., Maejima, Y., Mitsuda, K., Bradt, H. V., Remillard, R. A., Tuohy, I. R., Hoshi, R., \& Nakagawa, M. 1986, ApJ, 308, 635

Markoff, S., Falcke, H., \& Fender, R. 2001, A\&A, 372, L25

Markoff, S., \& Nowak, M. A. 2004, ApJ, 609, 972

Markwardt, C. B., \& Swank, J. H. 2003, Astron. Tel., 133

McClintock, J. E., \& Remillard, R. A. 2006, in Compact Stellar X-Ray Sources, ed. W. H. G. Lewin \& M. van der Klis (Cambridge: Cambridge Univ. Press), in press (astro-ph/0306213)

Miller, J. M., et al. 2004, ApJ, submitted (astro-ph/0406272)

Miyamoto, S., \& Kitamoto, S. 1989, Nature, 342, 773

Nowak, M. A., Vaughan, B. A., Wilms, J., Dove, J. B., \& Begelman, M. C. 1999, ApJ, 510, 874

Parmar, A. N., Kuulkers, E., Oosterbroek, T., Barr, P., Much, R., Orr, A., Williams, O. R., \& Winkler, C. 2003, A\&A, 411, L421

Pottschmidt, K. 2002, Ph.D. thesis, Univ. Tübingen

Revnivtsev, M. 2003, A\&A, 410, 865

Revnivtsev, M., Chernyakova, M., Capitanio, F., Westergaard, N. J., Shoenfelder, V., Gehrels, N., \& Winkler, C. 2003, Astron. Tel., 132

Reynolds, P. S. 1999, Astrophys. Lett. Commun., 38, 425

Rodriguez, J., Corbel, S., \& Tomsick, J. A. 2003, ApJ, 595, 1032

Rothschild, R. E., et al. 1998, ApJ, 496, 538

Rupen, M. P., Dhawan, V., \& Mioduszewski, A. J. 2003a, Astron. Tel., 210 2004a, Astron. Tel., 304

Rupen, M. P., Mioduszewski, A. J., \& Dhawan, V. 2003b, Astron. Tel., 139 2004b, BAAS, 8, 17.06 2004c, Astron. Tel., 314

Sobczak, G. J., McClintock, J. E., Remillard, R. A., Cui, W., Levine, A. M., Morgan, E. H., Orosz, J. A., \& Bailyn, C. D. 2000, ApJ, 544, 993

Steeghs, D., Miller, J. M., Kaplan, D., \& Rupen, M. 2003, Astron. Tel., 146

Sunyaev, R., \& Titarchuk, L. 1980, A\&A, 86, 121

Titarchuk, L., \& Fiorito, R. 2004, ApJ, 612, 988

Tomsick, J. A., Corbel, S., \& Kaaret, P. 2001, ApJ, 563, 229

Tomsick, J. A., \& Kaaret, P. 2000, ApJ, 537, 448

Tomsick, J. A., \& Kalemci, E. 2003, Astron. Tel., 198

Tomsick, J. A., Kalemci, E., Corbel, S., \& Kaaret, P. 2003, ApJ, 592, 1100

Varnière, P., Rodriguez, J., \& Tagger, M. 2002, A\&A, 387, 497

Vignarca, F., Migliari, S., Belloni, T., Psaltis, D., \& van der Klis, M. 2003, A\&A, 397, 729

Zdziarski, A. A., Poutanen, J., Paciesas, W. S., \& Wen, L. 2002, ApJ, 578, 357

Zhang, W., Jahoda, K., Swank, J. H., Morgan, E. H., \& Giles, A. B. 1995, ApJ, 449, 930 